\title{
Electroweak corrections to hadronic gauge boson production at large transverse momentum
}

\author{
A. Kulesza* \\ Deutsches Elektronen-Synchrotron DESY, Notkestrasse 85, \\ D-22607 Hamburg, Germany \\ E-mail: anna.kulesza@desy.de \\ M. Schulze \\ Institut für Theoretische Teilchenphysik, Universität Karlsruhe, \\ D-76128 Karlsruhe, Germany \\ E-mail: schulzeeparticle.uni-karlsruhe.de
}

We report on the calculations of the electroweak radiative correction to the transverse momentum distribution of gauge bosons $\left(W^{ \pm}, Z, \gamma\right)$ produced in association with a jet in hadronic collisions.

Numerical predictions for these processes at the LHC and Tevatron are discussed.

The 8th International Symposium on Radiative Corrections RADCOR

October 1-5, 2007

Florence, Italy

*Speaker. 
Gauge boson production belongs to the most important processes at hadron colliders. In particular, at the Large Hadron Collider (LHC) the $W$ and $Z$ boson, as well as direct photon production will be a benchmark process of great significance for e.g. the measurement of the parton distribution functions, measurement of the strong coupling constant $\alpha_{\mathrm{S}}$ or calibrating the response of the detectors. For the first time, it will be possible to produce gauge bosons in partonic collisions with the center-of-mass energy of a few $\mathrm{TeV}$, and consequently, explore production of gauge bosons with transverse momentum $\left(p_{\mathrm{T}}\right)$ in the $\mathrm{TeV}$ range. Given the relatively high cross sections for the leading-order $(\mathrm{LO}) p_{\mathrm{T}}$-distributions of gauge bosons, indicating small statistical errors of the future measurements, it is essential to improve the theoretical knowledge of these quantities by calculating higher-order radiative corrections. In the $\mathrm{TeV}$ regime, the logarithmic terms of the form $\alpha^{k} \log ^{2 k-i}\left(\hat{s} / M_{W}^{2}\right)(i \geq 0)$, known as the electroweak (EW) Sudakov logarithms, provide dominant contributions to the cross section and may well be responsible for corrections of several tens of percents. A survey of the literature on EW logarithms can be found in [1]. In particular, EW corrections to the hadronic $W$-boson production were studied in [2, 3, 牙, whereas the Z-boson and prompt photon production at large $p_{\mathrm{T}}$ were investigated in [5, 6, 7]. Numerical results for the one-loop corrections to the latter two processes can be also found in Ref. [8]. In the following we briefly report on the calculations of the gauge boson $(V)$ production in association with a jet $(j)$ in the process $p \stackrel{(-)}{p} \rightarrow V j$, presented in Refs. [2, 3, 6, 7].

\section{Calculation of the electroweak corrections}

The nature of the $\mathscr{O}(\alpha)$ corrections to the process $p \stackrel{(-)}{p} \rightarrow V j$, differs significantly between neutral $(V=Z, \gamma)$ and charged $\left(V=W^{+}, W^{-}\right)$gauge boson production. For the latter, the electromagnetic contributions cannot be separated from the weak ones in a gauge-invariant manner, hence making it necessary to include them in the calculations. In contrast to the corrections due to emission of massive gauge bosons, the contributions due to emission of real and virtual photons are separately singular and both have to be calculated. Effects due to real massive gauge boson emission are not included in our calculations of $W^{ \pm}, Z$ or $\gamma$ cross sections. ${ }^{1}$ For $W$-boson production we study the $p_{\mathrm{T}}$-distributions at the next-to-leading (NLO) order in $\alpha$ which at the partonic level are given by

$$
\frac{\mathrm{d} \hat{\sigma}^{a b \rightarrow W^{ \pm} k(\gamma)}}{\mathrm{d} p_{\mathrm{T}}}=\int \mathrm{d} \Phi_{2} \bar{\sum}\left|\mathscr{M}^{a b \rightarrow W^{ \pm} k}\right|^{2} F_{\mathrm{O}, 2}\left(\Phi_{2}^{W^{ \pm}}\right)+\int \mathrm{d} \Phi_{3} \bar{\sum}\left|\mathscr{M}^{a b \rightarrow W^{ \pm} k \gamma}\right|^{2} F_{\mathrm{O}, 3}\left(\Phi_{3}^{W^{ \pm}}\right) .
$$

In the calculations of $\mathscr{O}(\alpha)$ corrections to $p_{\mathrm{T}}$-distributions for neutral gauge boson $V^{0}=Z, \gamma$ only the weak contribution is considered and

$$
\frac{\mathrm{d} \hat{\sigma}^{a b \rightarrow V^{0} k(\gamma)}}{\mathrm{d} p_{\mathrm{T}}}=\int \mathrm{d} \Phi_{2} \bar{\sum}\left|\mathscr{M}^{a b \rightarrow V^{0} k}\right|^{2} F_{\mathrm{O}, 2}\left(\Phi_{2}^{V^{0}}\right) .
$$

$\Phi_{N}^{V}(N=2,3)$ above denotes the phase-space measure and $F_{\mathrm{O}, N}$ is the observable function

$$
F_{\mathrm{O}, N}\left(\Phi_{N}^{V}\right)=\delta\left(p_{\mathrm{T}}-p_{\mathrm{T}, V}\right) \boldsymbol{\theta}\left(p_{\mathrm{T}, j}-p_{\mathrm{T}, j}^{\min }\right) .
$$

\footnotetext{
${ }^{1}$ Such corrections to $p_{\mathrm{T}}$-distributions for $Z j$ and $\gamma j$ final states have been studied in [9].
} 
Our observable of interest is then the $p_{\mathrm{T}}$-distribution of a gauge boson produced in association with a jet carrying momentum $p_{\mathrm{T}, j} \geq p_{\mathrm{T}, j}^{\min }$. Note that for $W$-boson production, this is a more exclusive observable than that studied in [他. In particular, events with both $W$ and $\gamma$ at high $p_{\mathrm{T}}$ in the final state do not contribute to the cross section. Our calculations are LO in $\alpha_{\mathrm{S}}$ and the QCD corrections are not included.

The virtual corrections are calculated by reducing the one-loop diagrams to a minimal set of coupling structures, standard matrix elements and scalar integrals. The details of the calculation together with the discussion of the renormalization procedure and complete analytical results can be found in Refs. [3], [6], [7] for the $W$-, $Z$-boson and $\gamma$ production processes, respectively. For $W$-boson production, soft and collinear singularities due to virtual photons are regularized by either introducing small photon and fermion masses or using dimensional regularization. These singularities cancel against IR-singularities present in the real corrections. Two independent calculations combining real and virtual corrections are performed. They use the dipole subtraction formalism in the formulation which employs either dimensional regularization [11] or small photon and fermion masses [10] in order to regularize the singularities. The initial-state collinear singularities, remaining in the expressions for $W$ cross section after the subtraction procedure, are absorbed into the definition of the parton distribution functions (PDFs) using the $\overline{\mathrm{MS}}$ scheme with the scale $\mu_{\mathrm{QED}}=M_{W}$. The contribution with photons in the initial state is not included in this analysis of the $W$-boson production; they have been studied in [ 4 ]. Also in the case of $W$-boson production, the emission of photons from the final-state (anti-)quarks in processes of the type $q g \rightarrow W^{ \pm} q^{\prime} \gamma$ gives rise to collinear singularities. In order to avoid them we recombine the quark and photon momenta if the azimuthal-angle and pseudorapidity separation variable $R=\sqrt{\left(\eta_{q}-\eta_{\gamma}\right)^{2}+\left(\phi_{q}-\phi_{\gamma}\right)^{2}}$ is smaller than the separation parameter $R_{\text {sep }}$, i.e. $R<R_{\text {sep }}$. In this case we define $p_{\mathrm{T}, j} \equiv \sqrt{\left(\vec{p}_{\mathrm{T}, q}+\vec{p}_{\mathrm{T}, \gamma}\right)^{2}}$, otherwise $p_{\mathrm{T}, j} \equiv p_{\mathrm{T}, q}$ or $p_{\mathrm{T}, j} \equiv p_{\mathrm{T}, g}$ if the final-state particle $(k)$ is a gluon. As demonstrated in [3], different treatment of the quark and gluon final states leads to numerically irrelevant effects, therefore providing a viable approach to calculate predictions for our observable.

Apart from calculating the full $\mathscr{O}(\alpha)$ correction for the gauge boson production process, in [6, 7. 3] we also provide compact expressions for high-energy approximations of these results up to the next-to-next-to-leading logarithmic (NNLL) accuracy. In the high-energy regime the Sudakov logarithms are dominating terms in the one-loop corrections and the NNLL expressions deliver an excellent approximation of the full result. Moreover, we also calculate the next-to-leadinglogarithmic (NLL) approximation of the two-loop cross section. To illustrate the appearance of the Sudakov logarithms in the expressions for the $p \stackrel{(-)}{p} \rightarrow V j$ process, here we present this NLL asymptotic behaviour of the EW corrections for the specific case $V=W^{ \pm}$. For a discussion of the calculation we refer to [3]. The same class of corrections has been computed for Z-boson production in [5] and $\gamma$ production in [7]. The presented results are for the IR-finite part of the EW corrections, obtained after subtraction of IR singularities. As discussed in [3], at one loop this subtraction is performed in such a way that, to NLL accuracy, the IR-finite part corresponds to the complete EW correction regularized with a fictitious photon mass $M_{\gamma}=M_{W}$. The same prescription is adopted at the two-loop level.

The unpolarized squared matrix element for $\bar{q} q^{\prime} \rightarrow W^{ \pm} g$ process $^{2}$, including NLL terms up to

\footnotetext{
${ }^{2}$ Results for other partonic subprocesses contributing to $p \stackrel{(-)}{p} \rightarrow W^{ \pm} j$ are obtained via crossing and CP-symmetries.
} 
the two-loop level, has the general form

$$
\bar{\sum}\left|\mathscr{M}_{2}^{\bar{q} q^{\prime} \rightarrow W^{ \pm} g}\right|^{2}=64 \pi^{2} \alpha \alpha_{S} \frac{\hat{t}^{2}+\hat{u}^{2}}{\hat{t} \hat{u}}\left[A^{(0)}+\left(\frac{\alpha}{2 \pi}\right) A^{(1)}+\left(\frac{\alpha}{2 \pi}\right)^{2} A^{(2)}\right]
$$

with $\hat{t}=\left(p_{\bar{q}}-p_{W}\right)^{2}, \hat{u}=\left(p_{q^{\prime}}-p_{W}\right)^{2}$. The Born contribution reads $A^{(0)}=1 /\left(2 s_{\mathrm{W}}^{2}\right)$, with the shorthand $s_{\mathrm{W}}=\sin \theta_{W}$ for the weak mixing angle $\theta_{W}$. At one loop, the NLL part consists of double- and single-logarithmic terms and reads

$$
A^{(1)} \stackrel{N \mathrm{LL}}{=}-\frac{1}{2 s_{\mathrm{W}}^{2}}\left[C_{q_{\mathrm{L}}}^{\mathrm{ew}}\left(\mathrm{L}_{\hat{s}}^{2}-3 \mathrm{~L}_{\hat{s}}\right)+\frac{C_{\mathrm{A}}}{2 s_{\mathrm{W}}^{2}}\left(\mathrm{~L}_{\hat{t}}^{2}+\mathrm{L}_{\hat{u}}^{2}-\mathrm{L}_{\hat{s}}^{2}\right)\right] .
$$

Here we used the shorthand $\mathrm{L}_{\hat{r}}^{k}=\ln ^{k}\left(|\hat{r}| / M_{W}^{2}\right)$ for the logarithms and $C_{q_{\mathrm{L}}}^{\mathrm{ew}}=Y_{q_{\mathrm{L}}}^{2} /\left(4 c_{\mathrm{W}}^{2}\right)+C_{\mathrm{F}} / s_{\mathrm{W}}^{2}$, $C_{\mathrm{F}}=3 / 4, C_{\mathrm{A}}=2$. This expression is consistent with the process-independent results of [12] as well as with the NLL part of the one-loop asymptotic expressions presented in [3]. At two loops we obtain

$$
\begin{aligned}
& A^{(2) \stackrel{N \mathrm{LL}}{=}} \frac{1}{2 s_{\mathrm{W}}^{2}}\left\{\frac{1}{2}\left(C_{q_{\mathrm{L}}}^{\mathrm{ew}}+\frac{C_{\mathrm{A}}}{2 s_{\mathrm{W}}^{2}}\right)\left[C_{q_{\mathrm{L}}}^{\mathrm{ew}}\left(\mathrm{L}_{\hat{s}}^{4}-6 \mathrm{~L}_{\hat{s}}^{3}\right)+\frac{C_{\mathrm{A}}}{2 s_{\mathrm{W}}^{2}}\left(\mathrm{~L}_{\hat{t}}^{4}+\mathrm{L}_{\hat{u}}^{4}-\mathrm{L}_{\hat{s}}^{4}\right)\right]\right. \\
&\left.+\frac{1}{6}\left[\frac{b_{1}}{c_{\mathrm{W}}^{2}}\left(\frac{Y_{q_{\mathrm{L}}}}{2}\right)^{2}+\frac{b_{2}}{s_{\mathrm{W}}^{2}}\left(C_{\mathrm{F}}+\frac{C_{\mathrm{A}}}{2}\right)\right] \mathrm{L}_{\hat{s}}^{3}\right\},
\end{aligned}
$$

where $b_{1}=-41 /\left(6 c_{\mathrm{w}}^{2}\right)$ with $c_{\mathrm{W}}^{2}=1-s_{\mathrm{W}}^{2}$ and $b_{2}=19 /\left(6 s_{\mathrm{w}}^{2}\right)$ denote the one-loop $\beta$-function coefficients associated with the $\mathrm{U}(1)$ and $\mathrm{SU}(2)$ couplings, respectively.

\section{Numerical predictions}

The hadronic cross sections are obtained using LO MRST2001 PDFs $[14]^{3}$. We choose $p_{T}^{2}$ as the QCD factorization scale and, similarly, as the scale at which the running strong coupling constant is evaluated. We also adopt the value $\alpha_{S}\left(M_{Z}^{2}\right)=0.13$ and use the one-loop running expression for $\alpha_{S}\left(\mu^{2}\right)$, in accordance with the LO PDF extraction method of the MRST collaboration. For the exact values of the EW input parameters the reader is referred to [3, 6, 7]. The $W$-boson hadronic cross section was obtained with the following choices of the value of the cut on $p_{\mathrm{T}}$ of the jet: $p_{\mathrm{T}, j}^{\min }=100 \mathrm{GeV}$ for LHC and $p_{\mathrm{T}, j}^{\min }=50 \mathrm{GeV}$ for Tevatron. The value of the separation parameter below which the recombination procedure is applied was taken to be $R_{\text {sep }}=0.4$.

In Fig. 11 we present the relative NLO and NLL two-loop EW corrections to the $p_{\mathrm{T}}$-integrated cross sections for $W^{+}-, Z$-boson and $\gamma$ production in $p p$ collisions at $\sqrt{s}=14 \mathrm{TeV}$ (left column), as well as $W^{+(-)}, Z$ and $\gamma$ production in $p \bar{p}$ collisions at $\sqrt{s}=2 \mathrm{TeV}$ (right column). The corrections are shown as a function of $p_{\mathrm{T}}^{\mathrm{cut}}$, the lower limit of the integration of the distribution in $p_{\mathrm{T}}$. To underline the relevance of the corrections, we also plot an estimate of the statistical error, obtained assuming branching ratios $B R=2 / 9$ for $W$-boson production and $B R=0.306$ for $Z$-boson

\footnotetext{
${ }^{3}$ In order to consistently include $\mathscr{O}(\alpha)$ corrections in the calculation of hadronic $W$-boson production, PDFs used in the calculation should take into account QED evolution. The currently available NLO QED PDFs, i.e. MRST2004QED, include QCD effects at NLO. Since our calculation is LO in QCD we choose to use the LO QCD set (MRST2001) and take the QED factorization scale $\mu_{\mathrm{QED}}=M_{W}$ for which the neglected QED effects are small, as discussed in [3].
} 
production as well as asumming integrated luminosity of $300 \mathrm{fb}^{-1}$ for the LHC and $11 \mathrm{fb}^{-1}$ for the Tevatron. The NLO corrections are negative and grow in size with $p_{\mathrm{T}}$. They reach $-46 \%$ for $W^{+}$production and $-39 \%$ for $Z$ production at $p_{\mathrm{T}}^{\text {cut }}=2 \mathrm{TeV}$ at the LHC. Their size is much bigger than the corresponding statistical error for all values of $p_{\mathrm{T}}$ at the LHC. In the case of direct photon production at the LHC, the integrated EW corrections reach $-18 \%$ at $p_{\mathrm{T}}^{\text {cut }}=2 \mathrm{TeV}$, showing qualitatively the same behaviour wrt. the statistical error as observed for massive boson production. The contributions from the two-loop corrections (referred to in Fig. 11 as "NNLO") are positive. At $p_{\mathrm{T}}^{\text {cut }}=2 \mathrm{TeV}$ at the LHC they amount to $10 \%, 9 \%$ and $4 \%$ for the $W^{+}$-boson, $Z$-boson and $\gamma$ production, respectively. In terms of the estimated statistical error, these two-loop contributions amount to $1-3$ standard deviations for $p_{\mathrm{T}}$ of $\mathscr{O}(1 \mathrm{TeV})$. The NLO and NNLO EW corrections for $W^{-}$production at the LHC are quantitatively very similar to those for $W^{+}$production. The plots in the right column in Fig. 1 show results of the similar analysis for the gauge boson production at the Tevatron. The size of the NLO EW corrections is comparable with the expected statistical error for a significant range of $p_{\mathrm{T}}$ values of interest, the impact of the two-loop corrections appears negligible. These conclusions remain valid also for the smaller value of the integrated Tevatron luminosity, $\mathscr{L}=7 \mathrm{fb}^{-1}$.

Summarizing, at the LHC the NLO EW corrections to the process $p p \rightarrow V j$ where a gauge boson $V$ is produced with transverse momentum in the $\mathrm{TeV}$ range are large i.e. of the order of tens of percents. Their size is driven by the Sudakov logarithmic terms, dominating the one-loop virtual contributions. The two-loop NLL contributions are important for the correct interpretation of the results for the high- $p_{\mathrm{T}}$ gauge boson production at the LHC. At the Tevatron, the EW corrections are of lesser relevance, though the NLO EW corrections should be included in the analysis when considering precision measurements.

Acknowledgements: We are grateful to J. H. Kühn and S. Pozzorini for the collaboration on the project and to S. Dittmaier, B. Jäger and P. Uwer for helpful discussions. A.K. would like to thank the organizers for a very interesting and enjoyable conference.

\section{References}

[1] W. Hollik et al., Acta Phys. Polon. B 35 (2004) 2533 [hep-ph/0501246].

[2] J. H. Kühn, A. Kulesza, S. Pozzorini and M. Schulze, Phys. Lett. B 651 (2007) 160 [hep-ph/0703283].

[3] J. H. Kühn, A. Kulesza, S. Pozzorini and M. Schulze, arXiv:0708.0476 [hep-ph].

[4] W. Hollik, T. Kasprzik, B. A. Kniehl, Nucl. Phys. B 790 (2008) 138 [arXiv:0707.2553 [hep-ph]].

[5] J. H. Kühn, A. Kulesza, S. Pozzorini and M. Schulze, Phys. Lett. B 609 (2005) 277 [hep-ph/0408308].

[6] J. H. Kühn, A. Kulesza, S. Pozzorini and M. Schulze, Nucl. Phys. B 727 (2005) 368 [hep-ph/0507178].

[7] J. H. Kühn, A. Kulesza, S. Pozzorini and M. Schulze, JHEP 0603 (2006) 059 [hep-ph/0508253].

[8] E. Maina, S. Moretti and D. A. Ross, Phys. Lett. B 593 (2004) 143 [Erratum-ibid. B 614 (2005) 216] [hep-ph/0403050].

[9] U. Baur, Phys. Rev. D 75 (2007) 013005 [hep-ph/0611241].

[10] S. Dittmaier, Nucl. Phys. B 565 (2000) 69 [hep-ph/9904440]. 

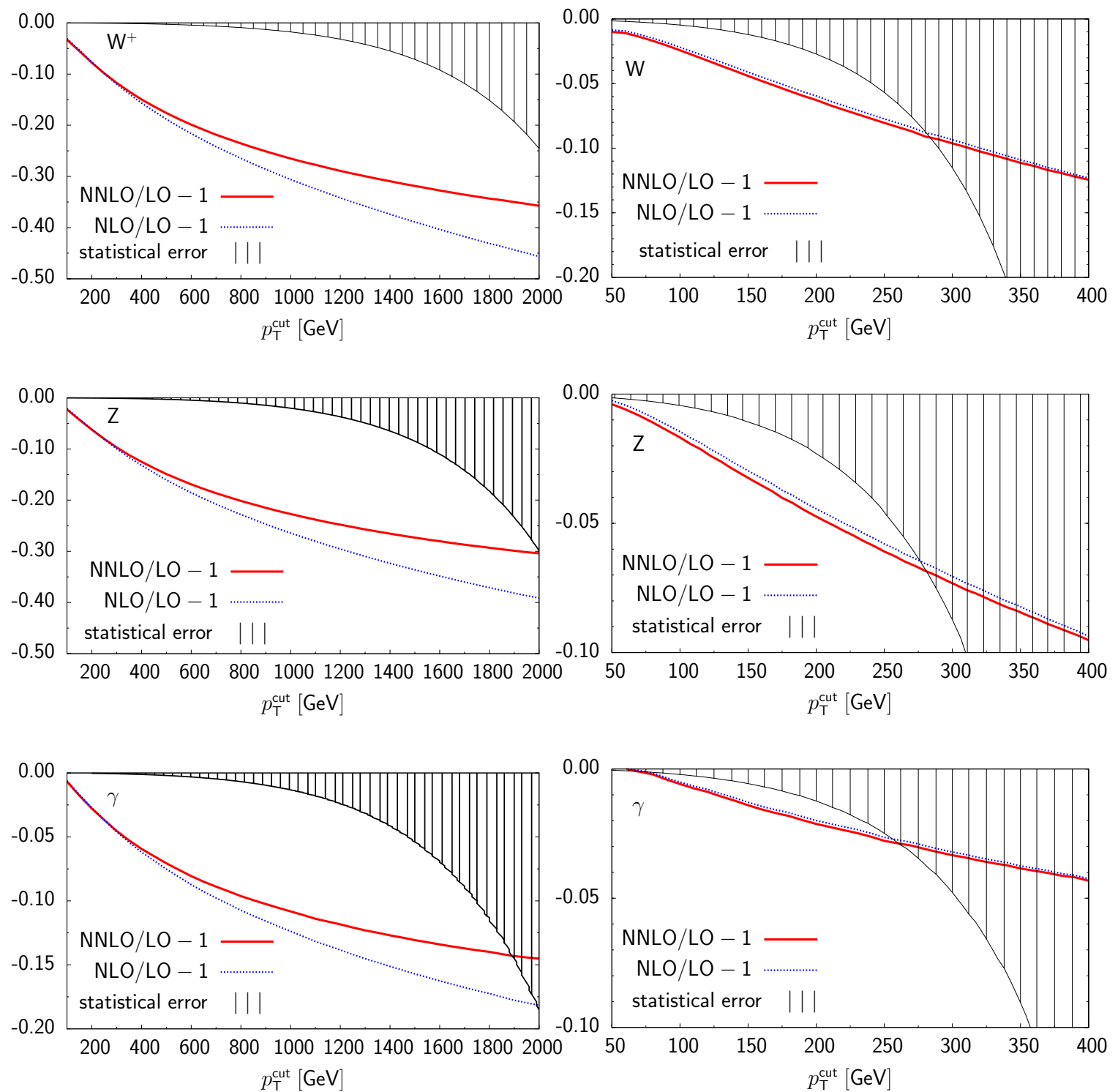

Figure 1: $\mathrm{NLO}$ and NNLO EW corrections to $p_{\mathrm{T}}$-integrated cross section for gauge boson production at hadron colliders versus estimated statistical error. From top to bottom, left column: predictions for $p p \rightarrow$ $W^{+} j, p p \rightarrow Z j, p p \rightarrow \gamma j$ at the LHC; right column: predictions for $p \bar{p} \rightarrow W j, p \bar{p} \rightarrow Z j, p \bar{p} \rightarrow \gamma j$ at the Tevatron.

[11] S. Catani and M. H. Seymour, Nucl. Phys. B 485 (1997) 291 [Erratum-ibid. B 510 (1998) 503] [hep-ph/9605323]; S. Catani, S. Dittmaier, M. H. Seymour and Z. Trocsanyi, Nucl. Phys. B 627 (2002) 189 [hep-ph/0201036].

[12] A. Denner and S. Pozzorini, Eur. Phys. J. C 18 (2001) 461 [hep-ph/0010201]; Eur. Phys. J. C 21 (2001) 63 [hep-ph/0104127].

[13] A. Denner, M. Melles and S. Pozzorini, Nucl. Phys. B 662 (2003) 299 [hep-ph/0301241].

[14] A. D. Martin, R. G. Roberts, W. J. Stirling and R. S. Thorne, Phys. Lett. B 531 (2002) 216 [hep-ph/0201127]. 